\title{
Marae, population et territoire aux îles de la Société. Le réseau māohi
}

\section{Claude Robineau}

\section{(2) OpenEdition}

1 Journals

Édition électronique

URL : http://journals.openedition.org/jso/5861

DOI : $10.4000 /$ jso.5861

ISSN : $1760-7256$

\section{Éditeur}

Société des océanistes

\section{Édition imprimée}

Date de publication : 30 juin 2009

Pagination : 79-90

ISBN : 978-2-85430-024-6

ISSN : 0300-953x

\section{Référence électronique}

Claude Robineau, «Marae, population et territoire aux îles de la Société. Le réseau māohi », Journal de la Société des Océanistes [En ligne], 128 | janvier-juin 2009, mis en ligne le 30 juin 2012, consulté le 10 décembre 2020. URL : http://journals.openedition.org/jso/5861 ; DOI : https://doi.org/10.4000/jso. 5861

\section{(c) $($ () $\ominus$}

Journal de la société des océanistes est mis à disposition selon les termes de la Licence Creative Commons Attribution - Pas d'Utilisation Commerciale - Pas de Modification 4.0 International. 


\section{Marae, population et territoire aux îles de la Société. Le réseau $m a \bar{a}$ ’ohi}

par

Claude ROBINEAU*

\section{RÉSUMÉ}

Le phénomène des marae aux îles de la Société, structures lithiques à caractère religieux, notable objet d'étude de José Garanger en Polynésie centrale, dont il fut avec Kenneth Emory et Yoshihiko Sinoto le grand spécialiste, pose, lorsqu'il est confronté aux récits tirés au XIX siècle de la tradition orale, le problème de la cohérence de l'organisation socio-politique dans l'aire culturelle de langue mā'ohi. Habituellement définie en termes de territoires rigoureusement bornés à la manière des États nationaux européens de la fin $d u X_{X I X}{ }^{e}$ et $d u$ ${ }_{X X}{ }^{e}$ siècle, elle devient, quand elle est évoquée en termes de marae, réseau de ces structures coagulant la vie économique et sociale et confronté sur un même territoire à des éléments d'autres réseaux voisins voire rivaux, pulvérisant du coup la notion d'un territoire socio-politique continu qu'évoque la métaphore du "pré-carré».

Mots-CLÉs : organisation socio-politique, chefferie, territoire, espace réticulaire, réseaux, marae, structure sociale

Cet article va, à nouveau, traiter des marae, ces " structures lithiques à caractère religieux ", selon la célèbre définition donnée par José Garanger à ce terme mä’ohi désignant ces édifices de Tahiti aux temps anciens, principalement rencontrés dans les îles de la Société qui constituent l'aire $m \bar{a}$ 'ohi et auxquelles on ajoute traditionnellement les Tuamotu de l'Ouest et les îles Australes.

\begin{abstract}
The phenomenon of the marae in the Society Islands, lithic structures with a religious function, a notable object of study by José Garanger in central Polynesia, of which he was a great specialist together with Kenneth Emory and Yoshihiko Sinoto, raises, when confronted with the accounts drawn at the 19th century from the oral tradition, the problem of the consistency of the socio-political organization in the cultural area of the mā'ohi language. Usually defined in terms of strictly limited territories as in the European national states of the end of $19^{\text {th }}$ and the $20^{\text {th }}$ century, it becomes, when referred in terms of marae, a network of these structures coagulating economic and social life and confronted on the same territory to elements of other close networks, sometimes rival, demolishing as a result the concept of a continuous socio-political territory that is enclosed in the "pré-carré" metaphor.
\end{abstract}

KEYWORDS: socio-political organization, chiefdom, territory, networks, marae, social structure

\section{L'œuvre de Garanger en Polynésie orientale}

La carrière de José Garanger peut être définie, grosso modo, par une première période de recherche exclusive de terrain - qui commença sur la Vaitepiha (district de Tautira, île de Tahiti) et se termina avec la découverte de Roy Mata (à Retoka, île d'Efate, Vanuatu) - et par un temps d'enseignement dont le début est symbolique-

* Directeur de recherche honoraire, IRD, 27, rue Castros, 33000 - Bordeaux. 
ment marqué par la collation du grade du doctorat d'État ès-Lettres suivie de l'accession au professorat des Universités au titre de l'Université de Paris I, temps intensément marqué par des recherches sur le terrain et notamment de retours fréquents en Polynésie orientale.

Pour José Garanger, la Polynésie orientale correspond géographiquement et historiquement au «triangle polynésien » Hawai'iNouvelle-Zélande-île de Pâques, laissant de côté Tonga, Samoa, Wallis-et-Futuna considérées comme la Polynésie occidentale (avec, à l'extrême ouest, les Polynesian Outliers) ; le centre du triangle (notamment à l'époque protohistorique) est formé des îles de la Société et des Marquises, constituant avec les Tuamotu, les Australes et les îles Cook une «Polynésie centrale ». C'est dire combien le phénomène marae occupa une place importante dans les recherches de José Garanger, avec des fouilles, des restitutions, une reconstruction de la vie sociale et qui s'étendit aux archipels polynésiens voisins. Ces recherches sont accessibles grâce aux publications auxquelles elles ont donné lieu, mais seulement pour une part, car d'autres informations, résultant notamment d'analyses comparatives, ont été livrées dans ses enseignements - nous en acquîmes seulement une partie en séminaire d'Océanie - et furent donc accessibles aux seuls étudiants qui les ont suivis.

Ce n'est pas le moindre des paradoxes que d'être conduit, pour l'explicitation des travaux de ce grand archéologue, à faire une archéohistoire des vestiges écrits, voire oraux, laissés par lui dans le corpus actuel de la connaissance archéo-historique des anciens Polynésiens. S'inspirant d'André Leroi-Gourhan et soucieux de replacer les découvertes archéologiques dans leur contexte humain, José Garanger a toujours privilégié l'écoute de la tradition orale susceptible à la fois de guider les fouilles archéologiques et d'en expliciter les résultats au plan historique et culturel. Je voudrais ici, pour ma part, prolonger en quelque sorte sa démarche en partant du phénomène des marae et montrer, concernant les plus importants d'entre eux, leur signification dans l'organisation de l'espace des anciens Mā'ohi.

\section{L'organisation de l'espace des anciens Mā'ohi}

Cette organisation est, notamment dans les îles de la Société, traditionnellement repérée (Henry, 1962 ; Handy, 1930 ; Ribourt, 1863) par les termes fenua «terre» et mata'eina'a étant traduit en anglais comme en français par le mot
« district ». Fenua désigne à la fois une île - île haute ou atoll - et une portion de terre, cette dernière généralement délimitée dans une île haute par l'espace compris entre deux crêtes radiales (issues d'un sommet de la chaîne centrale de l'île) et la section du littoral se trouvant entre les promontoires où les crêtes radiales viennent s'abîmer dans la mer (et, par extension, l'espace du lagon compris entre le littoral et le récif frangeant au droit des deux promontoires). Henry (1962 : 101) écrit ainsi à propos de Varari, Moorea :

" De Afa'a-tetea (pâle source fantôme) à Maenu (décoiffé) s'étend varari (endroit retiré et humide) dans le Nord-Ouest. La montagne qui domine est Tatiri (jette). Le terrain de réunion est Pa-hoa (l'enclos des amis) ; la pointe extérieure Nu'u-rua (deux armées) ; la rivière Tai-a-miti (mer vers l'extérieur); le marae Nu'urua : c'est un marae national qui existe encore; le mouillage pour petites embarcations est Tao-taha (sagaie oblique) et s'étend le long de la côte ouest... Le grand chef était Pu-nua-i-tera'i-atua (Conques au-dessus dans le ciel des Dieux). La maison 'arioi était Te-atea-pua (l'érythrina qui fleurit) et son chef Tohe'-ura (fondation rouge). »

Cette portion d'espace, terre et lagon correspondant, forme généralement une unité politique interne soumise à l'autorité d'un chef, ari'i. Cette unité est elle-même dépendante d'une entité plus large correspondant à un ensemble de vallées dominé par le chef de l'une d'entre elles qualifié du titre de prince, grand-chef, ari'i rahi (grand ari'i). À l'inverse, cette unité peut aussi être subdivisée en sous-unités correspondant à plusieurs vallées adjacentes ou secondaires, chacune étant gouvernée par un ari'i ri'i (" petit» ari'i). Mais une telle unité peut aussi constituer un élément d'une confédération d'entités, mouvante dans l'histoire : elle est présidée par le chef de l'une d'entre elles qui est qualifié de ari'i nui, très grand chef, chef suprême auquel les Européens, inspirés par les souvenirs de la Grèce ancienne, ont parfois donné le titre de roi.

À Tahiti (figure 1), il existe ainsi une vingtaine de ces unités appelées districts (fenua, mata'eina'a), dont le nombre varie selon les décomptes faits entre districts, grands districts, sous-districts et qui furent l'objet de regroupements mouvants entre Teaharoa (côte Est), Te-Porionuu (le Nord), Atehuru (la côte ouest), Teva-i-uta (les Teva-de-l'intérieur, la côte sud de Tahiti-nui), Teva-i-tai (les Teva-de-la-mer, ou Tahiti-iti, la presqu'île). À la fin du XvIII siècle, au moment de la « découverte », entre le passage de Bougainville (1768) et le premier voyage de Cook (1769), on a affaire à deux coalitions qui s'affrontent dans une bataille célèbre. 


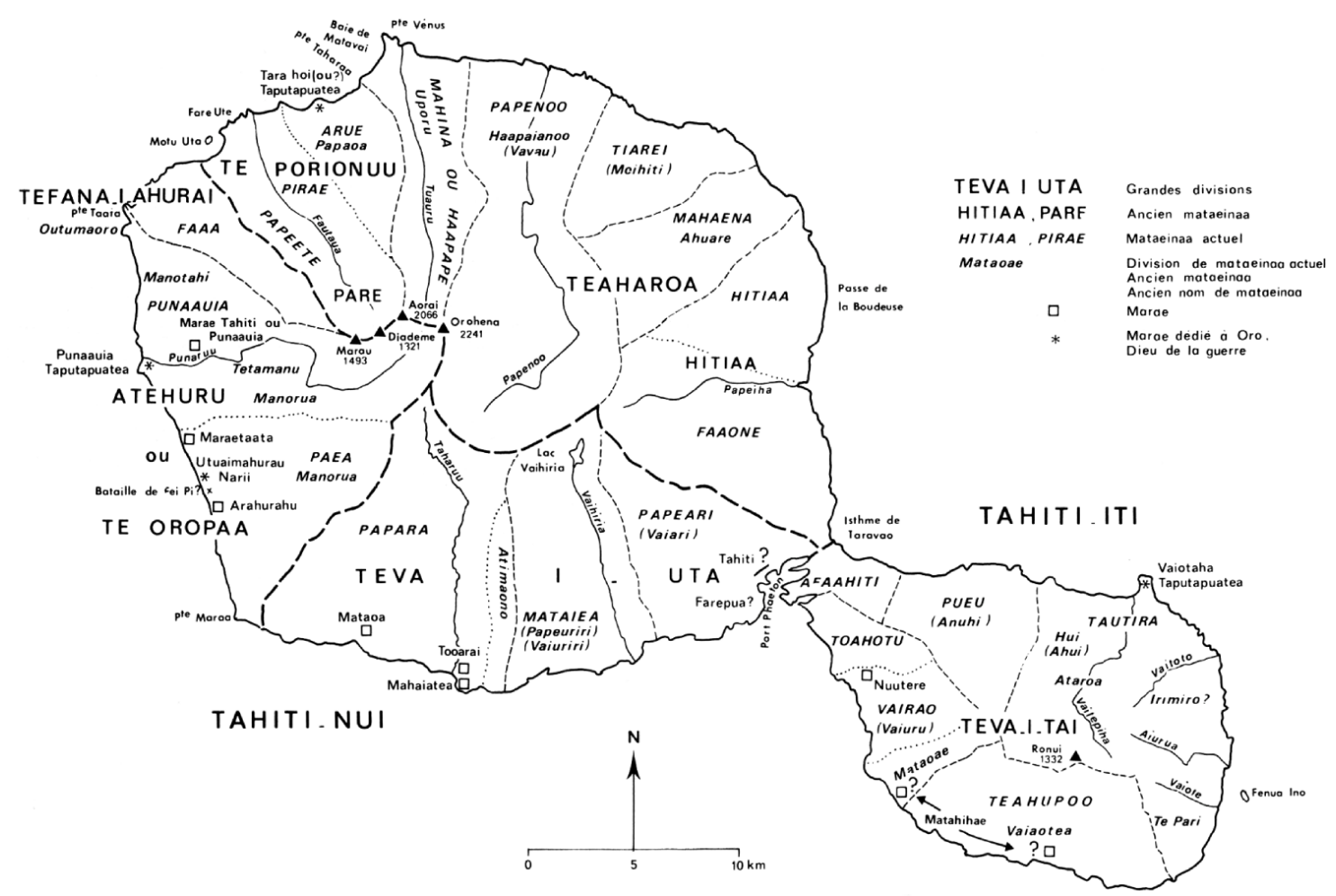

FIGURE 1. - Tahiti historique (carte d'après Handy [1930], Newbury [1967] et Garanger [1969])

Te-Porionuu, mené par Tutaha, grand guerrier de Pare, principale unité de cet ensemble et allié à Teva-i-tai, la presqu'île gouvernée par un chef appelé Vehiatua, affronte Teva-i-uta dont le chef est Amo, époux de la " reine Purea » et titulaire d'un symbole de suprématie, un maro'ura, ceinture de plumes rouges, qui lui donne le titre d'ari'i nui. Dans cette bataille, Amo est vaincu et Tutaha s'empare, pour le compte de son ari'i de Pare, le tout jeune Tu qui deviendra le chef Vairaatoa puis enfin Pomare I, du maro'ura détenu par Amo et du to'o, image du dieu 'Oro, alors principal dieu de Tahiti, qu'il dépose sur le marae Maraetaata dans le district de Paea, où Tu pourra revêtir le maro.

Selon certaines sources, ce marae serait celui de Tutaha qui est aussi dit chef de Paea ou d'Atahuru (ou peut-être Atehuru? cf. infra), ce qui n'est d'ailleurs pas la même chose, Paea n'étant qu'une fraction de Atahuru. C'est cette défaite de Papara qu'évoque Douglas Oliver (1974), qu'il date de 1768 et qui préfigure le transfert de la suprématie sur Tahiti, de Papara dont Amo est l'ari' $i$, à Pare, district des Pomare. Chose curieuse, la bataille qui se situe dans le district de Paea a lieu dans ce qui est considéré comme Atehuru ou Te-Oropaa, le domaine de Pohuetea, le grand-chef de la côte ouest, aux temps de Cook qui demeure neutre dans le conflit Pomare-Vehiatua/Amo-Purea. On retrouve six ou sept ans plus tard, à Tautira (presqu'île de Tahiti), le jeune Tu avec un Vehiatua du même âge et chef des Teva-de-la-mer, tous deux amis dans la compagnie de Maximo Rodriguez, interprète de la mission espagnole temporairement installée à Tahiti-iti (1774). J'ai le sentiment d'une certaine prééminence de $\mathrm{Tu}$ c'est Tu qui est chez Vehiatua et non le contraire - que pourrait confirmer la dévolution du domaine des Vehiatua aux Pomare quelques années plus tard avec l'extinction de cette lignée de Tautira.

\section{Organisation sociale et marae}

Les ari' $i$ disent (voir Teuira Henry, la princesse Ariitaimai, la reine Marau) tenir leur pouvoir des dieux. Selon Aurora Natua, dans l'ancienne structure sociale $m \bar{a}$ 'ohi, tout vient des dieux. Ils se projettent sur la terre par des marae et des $a h u$ qu'ils sont, selon les humains, censés fréquenter. On a ainsi la séquence : dieux $>$ marae $>$ ari' $i>$ sacré $\left(r a^{\prime} a\right)>$ autorité sur les profanes (noa), confortée par un réseau d'interdits (tapu qui a donné le mot «tabou») dont le non-respect entraîne la mort. Et ce n'est pas pour rien que Douglas Oliver fait partir sa description de l'Ancient Tahitian Society de ce qu'il appelle la Tahitian Cosmology, le monde tahitien des dieux. 
Le point d'articulation entre les dieux et les chefs (ari'i dotés de ra'a) est constitué par les marae. On ne reviendra pas sur la description de cet édifice lithique fondamental dans la vie des anciens Mā'ohi, renvoyant le lecteur à l'excellent petit livre de José Garanger, Pierres et rites sacrés (1969), rappelant seulement que le marae est le lieu privilégié de la manifestation des dieux, essentiellement le $a h u$ de pierres à l'une de ses extrémités et vers lequel il est orienté, et qu'il est un espace sacré $\left(r a^{\prime} a\right)$ et interdit aux êtres profanes.

Nous raisonnons ici sur les marae ari' $i$ ou marae royaux des chefs, grands chefs ari' $i$ maro'ura (ari'i à la ceinture rouge), mais nous savons (grâce à Henry) qu'il existait des marae correspondant aux différents niveaux sociaux, marae locaux ou mata'eina'a (de districts), marae "sociaux" dixit Henry, de va'a mata'eina'a, clan ou lignage, enfin des marae familiaux ou ancestraux, marae tupuna. Il existe aussi, correspondant à des groupes sociaux de spécialistes, des marae de docteurs, de constructeurs de pirogues, de pêcheurs. Il résulte du propos de Henry qui vient soit d'un texte écrit par le révérend Orsmond son grand-père soit d'informations recueillies par celui-ci de l'épouse tahitienne du missionnaire John Platt qui était cheffesse d'Uturoa à Raiatea, que l'existence des marae était un phénomène général de la société $m \bar{a}$ 'ohi, la majesté ou la discrétion des édifices se trouvant en rapport avec l'importance de la communauté concernée. C'est pourquoi, en se fondant sur cet écrit et sur l'évidence de la proximité nécessaire d'un lieu d'approche des dieux dans une population aussi croyante et aussi dépendante de la consultation de ses dieux que l'étaient les Mā'ohi pour tous les actes de leur vie, Oliver imagine l'existence, à proximité de la résidence des moindres segments de parenté, de marae très simples concernant ce qu'il appelle des kin-congregations, soit en langage non connoté par les usages anglo-saxons protestants, des communautés de droit de fidèles, parents ou apparentés.

\section{Marae et terres}

Locaux, sociaux ou familiaux, les marae sont, pour leurs titulaires ari'i chefs de clans ou chefs de familles, les points d'ancrage sur la terre dont l'usage leur est dévolu par leur fonction. La possession de la terre n'est pas individuelle mais collective, se décomposant en deux droits, un droit éminent qui appartient à la collectivité concernée et un droit d'usage détenu par le chef de cette collectivité qui, peu à peu, va en concé- der à son tour l'usage aux groupes et à leurs chefs qui se trouvent placés sous sa mouvance, et ainsi de suite. On a donc affaire à un emboîtement de droits qui correspond à l'emboîtement des groupes humains, lesquels droits se trouvent symbolisés à chaque niveau par un marae qui est celui du groupe concerné et dont le chef porte le nom d'un titre attaché au marae. C'est la raison pour laquelle, jusqu'à la fin du XIX ${ }^{\mathrm{e}}$ siècle, avant que la propriété des terres ne soit enregistrée, cette propriété était transmise par la descendance généalogique de l'ancêtre titulaire du marae dont la terre dépendait.

Ainsi, la chefferie de Varari décrite supra a pour chef un ari'i qui porte le titre de Punua-ite-rai-atua simplifié en Punuateraitua, nom du titre accroché au marae Nuurua dit «national». Dans le texte de Teuira Henry, l'influence du marae ari'i de Varari dépasse aussi les limites du district, englobant au moins les autres districts de la côte sud-ouest de Moorea, Moruu, Haapiti, Atiha (figure 2).

Ariitaimai, membre de la famille Marama qui gouvernait Haapiti, décrit dans ses Mémoires, rédigés par Henry Adams (1964), l'ascension des chefs de Haapiti avec la conduite pusillanime des chefs de Varari qui, en dépit de leur titre sur le marae Nuurua, ne soutiennent pas le prestige attendu du port d'un tel titre. La princesse raconte comment son ancêtre Marama, ayant acquis de facto un prestige qui dépassait sa chefferie de Haapiti, chercha à en acquérir une reconnaissance de jure, par des droits sur le marae prestigieux, en édifiant à son nom une structure dans l'enceinte de Nuurua ou à proximité de cette dernière et donc en plein milieu du district de son prestigieux rival.

Par la suite, Marama maria son fils à la fille de Punuateraitua ari'i de Varari et aurait acquis ainsi des droits sur le grand marae Nuurua (TeriiVahine-i-te-tauo-o-te-rai, 1970). Le même auteur nous signale qu'un Marama ultérieur se maria avec une petite cousine de Amo, chef de la branche aînée des Teva de Papara, Terai-efa-iMaraetaata, dont le nom indique une connexion avec le marae éponyme de Paea à Tahiti. Leur fils fut Taipoto que les missionnaires disent « chef de Moorea » (Davies, 1961 : 116).

Un autre Punuateraitua fut Mahine, grand guerrier de Moorea qui aurait pu relever le prestige des ari'i de Varari et ainsi reléguer à un rang secondaire les Marama qui n'étaient pas directement connectés à Nuurua. Mais, il mourut trop tôt pour que ses succès aient pu bénéficier à sa famille de Varari et mettre un terme à la progression des Marama. 


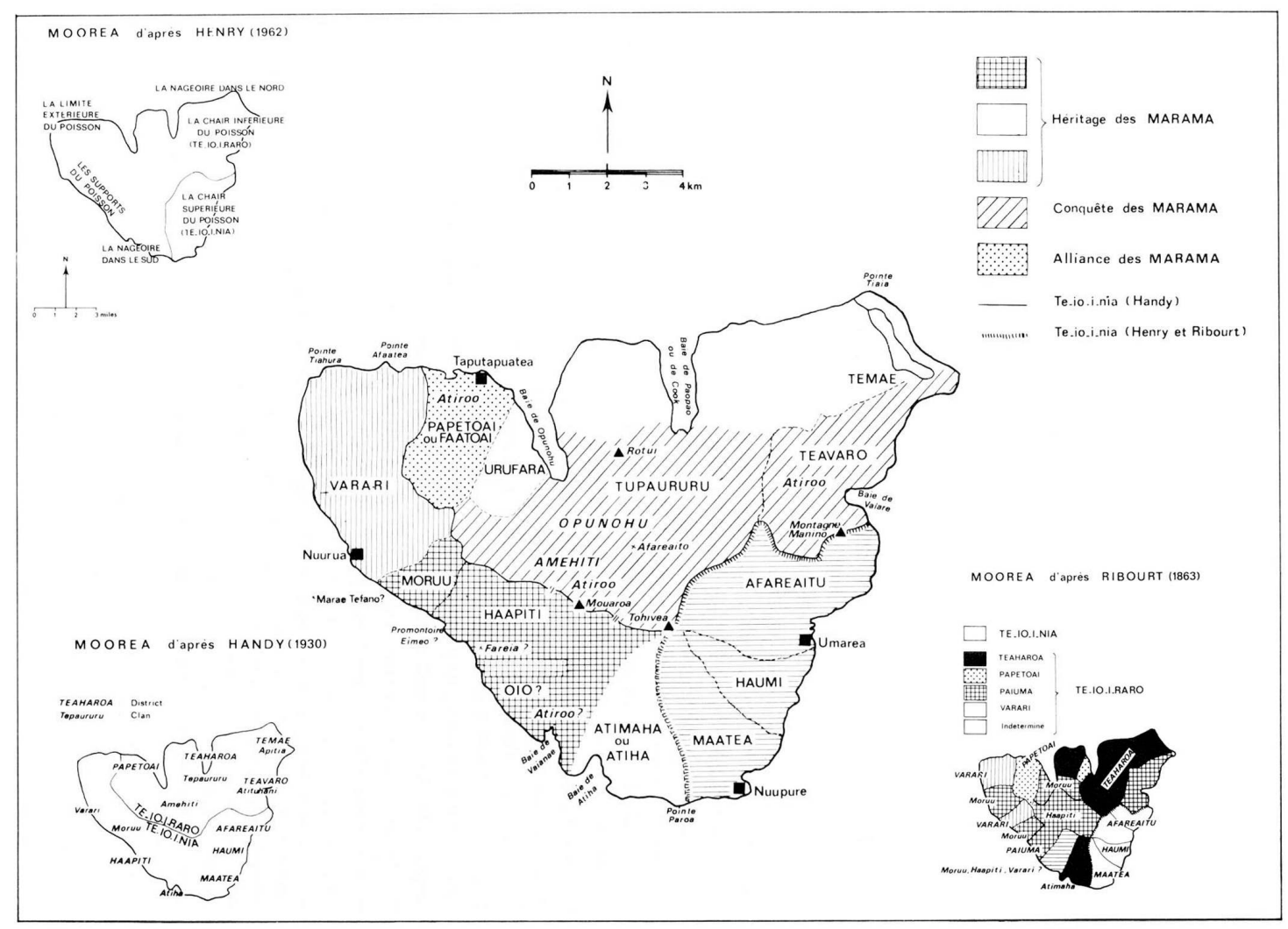

FiguRE 2. - Moorea ancien (carte d'après Ribourt [1863], Handy [1930] et Henry [1962])

\section{Enchevêtrement de territoires et ambiguïtés de leurs limites}

Les cartes de Tahiti et Moorea (figures 1 et 2) et les tableaux (1 et 2) portant sur Tahiti montrent, selon les principales sources disponibles (Henry, 1962 ; Ribourt, 1863 ; Handy, 1930), la localisation des principaux districts de ces îles, terres (fenиa) bien délimitées (d'un sommet de la chaîne axiale, entre deux crêtes radiales délimitant une portion de littoral et de lagon) et comportant un contenu humain (mata'eina'a) articulé en clans (va'a mata'eina'a), lignages ('ati) et familles (feti'i, les parents ; 'opu, la descendance d'un couple; 'opu hoe, le groupe des frères et sœurs) : une hiérarchie de groupes représentés par leurs chefs et symbolisés par une hiérarchie de marae rattachés les uns aux autres par le principe de la pierre de fondation.

On a, en fait, affaire à une vision européenne de "prés-carrés ", limités par des frontières, emboîtés les uns dans les autres à l'image de l'époque féodale et seigneuriale du Moyen-Âge européen. Les relations inter-politiques entre ces unités étaient faites d'alliances et de contrealliances à propos desquelles Adams s'égare dans des comparaisons historiques européennes qui n'ont aucun sens à Tahiti parce qu'il raisonne comme si ces unités étaient des États fondés sur des différences sensibles de langue, de culture et de tradition historique... Bien que l'on soit depuis longtemps revenu des excès engendrés par cette vision, l'image d'espaces territoriaux délimités bien distincts demeure. C'est la raison pour laquelle les faits relevés tant à Varari (Moorea) qu'à Paea (Tahiti) posent interrogation : à Moorea, c'est le ari'i de Haapiti dont le prestige dépasse l'horizon de son district qui vient édifier à Varari, dans le district de son rival Punuateraitu près du marae Nuurua dont ce dernier est le titulaire, une petite structure annexe qui, sans lui donner le titre sur le marae principal, peut lui permettre de participer à sa renommée. À Tahiti, on peut s'interroger à propos du marae Maraetaata dans le district de Paea qui passe pour être le marae de Tutaha, grand guerrier du district de Pare, et, plus encore, sur le fait que le combat qui, en 1768, oppose le Nord et la presqu'île contre le Sud se déroule sur un district, Paea, qui fait partie de la confédération de l'Ouest, distincte des trois autres et dont aucune information ne montre qu'elle est impliquée dans ce conflit, pour ou contre l'un ou l'autre camp. 


\begin{tabular}{|c|c|c|c|c|c|c|}
\hline RODRIGUEZ & MORRISON & WILSON & ORSMOND/HENRY & RIBOURT & MARAU/HANDY & OLIVER \\
\hline $\begin{array}{c}\text { Vehiatua (1) } \\
\text { Oyari (Vaiari) }\end{array}$ & $\begin{array}{l}\text { Tetevaiuta } \\
\text { Vaiari }\end{array}$ & $\begin{array}{c}\text { Tedevvaruta } \\
\text { wyere }\end{array}$ & $\begin{array}{l}\text { Tahiti-nui } \\
\text { Papeari ou Vaiari }\end{array}$ & $\begin{array}{l}\text { Tevaiuta } \\
\text { Hoo Matavana (4) }\end{array}$ & $\begin{array}{c}\text { Teva-i-uta } \\
\text { Papeari (Vaiari) }\end{array}$ & $\begin{array}{l}\text { Teva i uta } \\
\text { Vaiari }\end{array}$ \\
\hline Vaiuriri & Vaiuriri & Wyereede & $\begin{array}{l}\text { Papeuriri ou Vaiuriri } \\
\text { ou Mataiea }\end{array}$ & Mataiea $\begin{array}{l}\text { Papeuriri } \\
\text { Mairipeehe }\end{array}$ & Mataiea & Vaiuriri \\
\hline $\begin{array}{l}\text { Atimaono } \\
\text { Papara }\end{array}$ & Papara & $\begin{array}{l}\text { Attemone } \\
\text { Papara } \\
\text { Ahaheinah (Vainiania) }\end{array}$ & $\begin{array}{l}\text { Atimaono } \\
\text { Papara }\end{array}$ & $\begin{array}{l}\text { Atimaono } \\
\text { Papara }\end{array}$ & $\begin{array}{l}\text { Atimaono } \\
\text { Papara }\end{array}$ & $\begin{array}{l}\text { Atimaono } \\
\text { Papara }\end{array}$ \\
\hline Ohitia (Hitiaa) (2) & $\begin{array}{l}\text { Teporionuu et Teaharoa } \\
\text { Hitiaa }\end{array}$ & Hedeah & Hitiaa & $\begin{array}{l}\text { Teaharoa } \\
\text { Taero (Hitiaa) (4) }\end{array}$ & $\begin{array}{l}\text { Aharoa } \\
\text { Hitiaa }\end{array}$ & $\begin{array}{l}\text { Te Aharoa } \\
\text { Hitiaa }\end{array}$ \\
\hline $\begin{array}{l}\text { Tu et ses alliances (3) } \\
\text { Ohaena (Mahaena) }\end{array}$ & & $\begin{array}{l}\text { Tepirreonoo } \\
\text { Waaheina }\end{array}$ & Mahaena & Ahuare (Mahaena) (4) & Mahaena & Mahaena \\
\hline Otiarei & Tiarei & Otyare & Tiarei & $\begin{array}{l}\text { Te Meheti)(Tiarei) } \\
\text { Te Ne }\end{array}$ & Tiarei (Meihiti) & Tiarei \\
\hline Nonohea (Onohea) & Onohea (Onofea) & $\begin{array}{l}\text { Nanoonanoo } \\
\text { Honoowhia } \\
\text { Hababoonea } \\
\text { Hervow } \\
\text { Wharoomy }\end{array}$ & & & & \\
\hline Apayano & Haapaianoo & $\begin{array}{l}\text { Whapiano } \\
\text { Whyruppo (Faaripo) }\end{array}$ & Papenoo $\mid \begin{array}{l}\text { Intérieur: } \\
\text { Te-piha-ia } \\
\text { Teta }\end{array}$ & $\begin{array}{c}\text { Haururu (Papenoo) } \\
(4)\end{array}$ & $\begin{array}{l}\text { Papenoo } \\
\text { (Vavau) }\end{array} \mid \begin{array}{l}\text { Intérieur: } \\
\text { Hapaianoo }\end{array}$ & Haapaianoo \\
\hline $\begin{array}{l}\text { Matabay (Matavai) } \\
\text { Opare }\end{array}$ & $\begin{array}{l}\text { Matavai } \\
\text { Pare }\end{array}$ & $\begin{array}{l}\text { Matavai } \\
\text { Oparre }\end{array}$ & $\begin{array}{l}\text { Haapape (Uporu) } \\
\text { Teporionuu Arue } \\
\text { Pare }\end{array}$ & $\begin{array}{l}\text { Mahina (5) } \\
\text { Te Porionuu Arue } \\
\begin{array}{l}\text { Pare } \\
\text { Pan }\end{array}\end{array}$ & $\begin{array}{l}\text { Haapape (Uporu) } \\
\text { Porionuu Arue } \\
\text { Pare }\end{array}$ & $\begin{array}{l}\text { Te Porionuи } \\
\text { Haapape } \\
\text { Arue } \\
\text { Pare }\end{array}$ \\
\hline Tetaha & Tetaha & Tettaha & Faaa & $\begin{array}{l}\text { Tefana iahurai } \\
\text { Faaa } \\
\text { Te Oropaa }\end{array}$ & $\begin{array}{c}\text { Oropaa } \\
\text { Faaa (Tefana) }\end{array}$ & $\begin{array}{l}\text { Te Fana } \\
\text { Faaa } \\
\text { Te Oropaa }\end{array}$ \\
\hline $\begin{array}{l}\text { Tepunaauia } \\
\text { Atehuru }\end{array}$ & Atahuru & Attahooroo & Teoropaa $\begin{array}{l}\begin{array}{l}\text { Manotahi } \\
\text { (Punaauia) }\end{array} \\
\begin{array}{l}\text { Manorua } \\
\text { (Paea) }\end{array}\end{array}$ & $\begin{array}{l}\text { Manotahi (Punaauia) } \\
\text { Manorua (Paea) }\end{array}$ & \begin{tabular}{l|l} 
Punaauial Intérieur: \\
Peaea
\end{tabular} & $\begin{array}{l}\text { Punaauia } \\
\text { Paea }\end{array}$ \\
\hline
\end{tabular}

Notes: (1) Suprématie de Vehiatua II ; (2) District indépendant; (3) Districts de Tu (Pomare I) et de ses parents et alliés; (4) Districts identifiés par leurs limites; Hoo Matavana: plutôt Hooma tavana (MARAU, 1971, p. 83); (5) Nom moderne de Haapape. Sources: cf. tableau Tahiti-iti.

TABlEAU 1. - Districts de Tahiti à la fin du XVIII ${ }^{\mathrm{e}}$ siècle : Tahiti-nui

\begin{tabular}{|c|c|c|c|c|c|c|c|}
\hline RODRIGUEZ & MORRISON & WILSON & ORSMOND/HENRY & \multicolumn{2}{|c|}{ RIBOURT } & MARAU/HANDY & OLIVER \\
\hline Vehiatua (1) & Tahiti-iti & Tiarraboo & Taiarapu & Tevaitai & & Teva-i-tai (Taiarapu) & Teva i tai \\
\hline Afaahiti & Afaahiti & Owaheite & Afaahiti & Taahiti (2) & Faahiti (3) & Afaahiti & Afaahiti \\
\hline Anuhi & & Ahnooe & Pueu ou Anuhi & Anuhi ou & Pueu (2) (3) & Pueu & Pueu \\
\hline Ahui [à Vaiaotea] & & Ahooe & & & & Hui & \\
\hline Ohatutira & $\begin{array}{l}\text { Tautira (Oheitepeha, } \\
\text { Vaitepiha) }\end{array}$ & Owahie & Tautira ou Fatutira & & Tautira (3) & Tautira & Tautira \\
\hline Ataroa, vallée & & Attaroa & & & & & \\
\hline de Tautira & & Touttera (Tautira) & & & & & \\
\hline Irimiro (?) & & & & & & & \\
\hline Guayurua (Vaiurua, & & & & Taiarapu & & & \\
\hline Aiurua) (?) & & & & (2) & & & \\
\hline & & Wyyote (Vaiote) & & & & & \\
\hline Tepari & Tepari & Tehappea (Taapea) & & & & & \\
\hline Tayarabu (Taiarapu)? & & Ohotaboo? & & & & & \\
\hline Oyautea (Vaiaotea) & Vaiaotea & Wiyouea & Teahupoo & & Teahupoo (3 & ) Teahupoo & Teahupoo \\
\hline Mataoae & Mataoae & Matawei & Mataoae & Mataoae (2) & Mataoae (3) & & Mataoae \\
\hline Vaiuru & Vaiuru & Wyooroo & $\begin{array}{l}\text { Vairao (Vaiuru ou } \\
\text { Papeuru) }\end{array}$ & Vairao (2) & Vairao (3) & Vairao & Vairao \\
\hline & & Toahoutou & Toahotu & & Toahotu (3) & & Toahotu \\
\hline
\end{tabular}

Notes: (1) Districts placés sous la suprématie de Vehiatua II ; (2) Anciens districts, chefferies; (3) Districts existants au milieu du XIX" siècle. Sources: RODRIGUEZ, 1930, Journal (dépouillement personnel). - MORRISON, 1966, Journal, pp. 135-136. - WILSON, 1799, A Missionary Voyage.... carte pp. 184-185 (carte du cap.
Cook mise à jour par J. Wilson). - ORSMOND-HENRY, in HENRY, 1962, pp. 76 et suiv. - RIBOURT, 1863, État de l'lle Taüti... pp. 305-310. - MARAU/HANDY, in HANDY, 1930, p. 45 (carte). - OLIVER, 1974, II, p. 974 (carte).

Tableau 2. - Districts de Tahiti à la fin du XviII ${ }^{\mathrm{e}}$ siècle : Tahiti-iti

Dans ces cas, les limites ne comptent pas. Il est possible que Varari ait donné son accord pour que son voisin édifie chez lui, à côté du grand marae Nuurua, ce Nuurua bis. Il est envisageable que, par ses relations de parenté, Tutaha ait pu avoir un marae à Paea et, peut-être encore, y a-t-il eu à propos du conflit de Pare et de la Presqu'île contre Papara une déclaration de neutralité de Pohuetua, grand-chef de l'Ouest (Punaauia et Paea), acceptant que les protagonistes se rencontrent sur son propre terrain à mi-chemin de leur territoires respectifs. On sait bien que, dans les temps anciens, l'intérieur de Tahiti était peuplé. Des récits font état, lors des guerres, de cheminements ou de retraites par l'intérieur (encore que ces passages dans la montagne ne soient pas des plus faciles). Il y avait aussi la possibilité de naviguer, mais il fallait trouver un lieu de débarquement, ce qui n'était guère aisé, le littoral étant très occupé et pas forcément par des amis. On a ainsi du mal à saisir comment les guerriers de la Presqu'île, qui sont à 
l'est des districts de la côte sud, ont pu, avec leurs amis du Nord, livrer bataille à ces districts de la côte sud en un lieu qui se trouve au nord-ouest de ces derniers, sauf à admettre que les districts en forme de "prés-carrés » sont une fable, qu'ils représentent non un territoire compact mais des zones de concentrations de lieux spécifiques, marae, terrain de réunion, maison 'arioi, grève, terre de cultures, qui constituent le domaine d'un chef, celui-ci ayant généalogiquement autorité sur des chefs inférieurs disposant de domaines semblables, l'ensemble formant un réseau et le district se trouvant constitué d'un entremêlement de réseaux liés par les règles de parenté mä’ohi.

\section{Réseaux et espaces réticulés}

On sait que de telles organisations d'espaces « réticulés » existent en Nouvelle-Calédonie et coexistent au Vanuatu avec des systèmes de chefferies analogues au modèle tahitien. Jean Guiart (1963) montre qu'en Nouvelle-Calédonie, les unités sociales s'édifient à partir d'un tertre, lieu privilégié d'espace de parenté, se développent de tertre en tertre à partir de ce tertre-souche et selon les règles de parenté, construisant en guise de territoire un réseau de lignes unissant les tertres apparentés et entrecroisant dans l'espace d'autres réseaux correspondants à d'autres unités sociales. Au Vanuatu, Joël Bonnemaison (1996) caractérise la géographie culturelle des Nouvelles-Hébrides comme un espace réticulaire qu'il définit, en s'inspirant de Bensa et Rivierre (1982), comme :

" un chaînage de lieux qui sont autant des "chemins d'alliance"que des territoires se succédant le long d'un itinéraire. Les groupes mélanésiens ne sont pas des groupes clos par une "frontière" [des "prés-carrés" dit-il ailleurs], mais des réseaux de petites sociétés locales en communication constante les unes avec les autres selon leurs relations de proximité. »

D'autres éléments recueillis dans le monde $m \bar{a}$ 'ohi peuvent être versés au dossier. Le premier est tiré de la nomenclature des districts relevée dans les années 1840 pour le compte du gouverneur Bruat par le capitaine Ribourt qui, à Moorea, localise à plusieurs endroits de l'île des annexes d'un même district. Sur la carte de Moorea (figure 2), cela concerne Papetoai, Moruu, Varari. Différentes hypothèses peuvent alors être proposées :

- des migrations de populations relevant d'un même chef et s'installant à différents endroits ;
- un manque de terres dans le district principal en raison de l'accroissement de la population nécessitant la migration dans des zones vides ;

- des relations matrimoniales apportant au chef A épousant la femme chef B des terres de la chefferie $\mathrm{B}$ qui, à la génération suivante iront au descendant de $\mathrm{A}$ et $\mathrm{B}$ titulaire par héritage de la chefferie A ;

- ou encore, pour ces districts à localisation multiple, des chefferies en réseaux de lieux plutôt qu'en territoire compact et cerné.

Remarquons aussi que lorsque Ribourt a mené son inventaire des chefferies, il n'a pas fait œuvre de géomètre arpenteur. Il a dû procéder à des enquêtes auprès des chefs concernés, les interrogeant sur la consistance et les limites de leurs districts, avec très vraisemblablement à l'esprit l'image de territoires continus cernés et bordés par les limites énoncées, ainsi que les décrit d'ailleurs Tahiti aux temps anciens dont les rédacteurs européens ou européanisés devaient avoir une conception semblable de la notion de territoire. Cette vision préconçue a pu l'empêcher de poser les bonnes questions. Si l'on se rapporte au lien existant, à travers la notion de marae ari' $i$, entre peuplement, unité politique et marae, il conviendrait peut-être de considérer une unité politique telle qu'une chefferie comme un réseau de marae affiliés, éventuellement concurrencés sur des sites proches, plutôt que comme un territoire circonscrit dont l'intérieur est parallèlement hiérarchisé en unités sociales de plus en plus localisées, les marae s'y rapportant étant pareillement affiliés (par le rite de la pierre de fondation), les terres relevant de ces unités étant symboliquement rattachés aux marae correspondants.

Un autre élément peut être tiré de la structure socio-politique ancienne de Huahine aux îles Sous-le-Vent (figure 3). Cette île, actuellement divisée en huit districts (devenus "sections de communes » depuis la réforme municipale des années 1970), était, selon les traditions recueillies tant par Handy (1930) que par Emory (1932), organisée différemment des autres îles de la Société. Les marae royaux (ari'i) des différents districts (mata'eina'a) se trouvaient tous rassemblés en un même lieu, à Maeva, avec au-dessus d'eux (métaphoriquement mais aussi physiquement) un marae dit «national » Matairea rahi, tandis que les marae secondaires (des groupes dépendant des ari'i) se trouvaient disséminés dans les deux moitiés de Huahine, mais sans que l'on puisse dégager pour les mata'eina'a correspondants des territoires distincts (figure 3). 


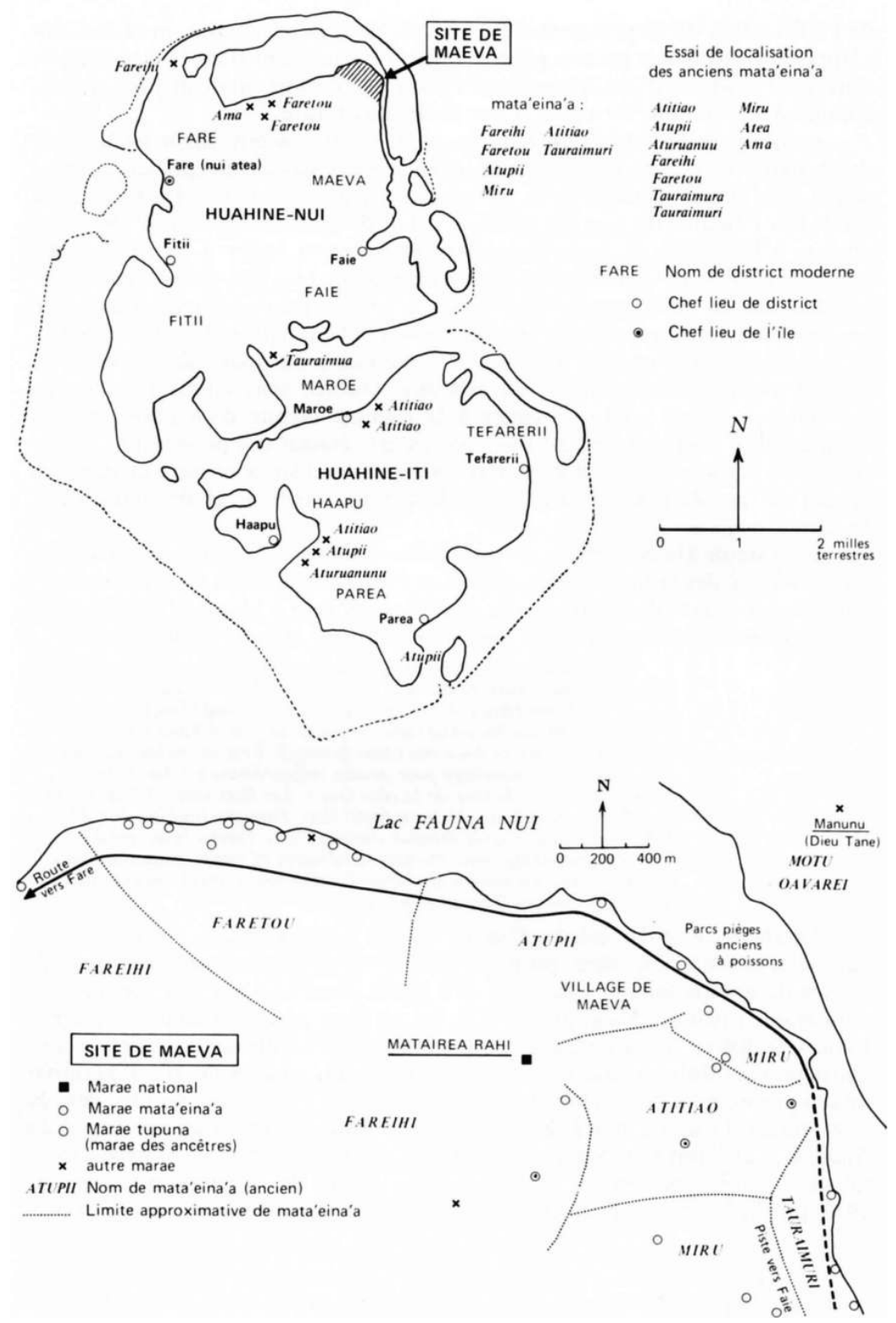

Figure 3. - Carte de l'île de Huahine et site de Maeva

Un tel dispositif suggère que chaque chefferie formait un mata'eina'a qui, par accroissement démographique, a pu disséminer ses établissements sur les terres disponibles sans que l'organisation sociale se trouve contrariée par l'organisation territoriale résultant de la progression $\mathrm{du}$ peuplement et sans qu'on en arrive à une territorialisation de facto des mata'eina'a aboutissant à la délimitation d'un territoire propre à chacun d'eux. La division territoriale de Huahine en huit districts, qui apparaît à la fin du $\mathrm{XIX}^{\mathrm{e}}$ siècle et que la colonisation reprend à son compte, peut résulter de l'action des missionnaires de la LMS (London Missionary Society) soucieux d'édifier des stations rassemblant la population en quelques lieux privilégiés pour faciliter la prédication et protéger leurs fidèles dans une époque troublée. La convention signée pour la France à Londres par le comte de Jarnac en 1847 prévoyait en effet que les îles Sous-le-Vent ne faisaient pas partie du protectorat français établi sur les États de la reine Pomare Vahine IV, demeuraient indépendantes et l'histoire politique de Huahine fut donc, par la suite, particulièrement mouvementée.

Le décollement de la notion de mata'eina'a, traduit par district, d'avec la signification territoriale habituelle franco-anglaise accordée à ce dernier terme renvoie aux doublets mä'ohi fenualmata'eina'a, tufa'alva'a mata'eina'a (clan), patul'ati (lignage) qui expriment l'adéquation socio-économique du groupe sur une terre, sans 
que cela implique une confusion entre ces espaces socio-économiques (et au niveau supérieur socio-politiques) et un espace territorial. La distinction sémantique entre fепиа (la terre) et mata'eina'a (son contenu humain) veut montrer qu'à la différence de la conception historique européenne territoriale de l'autorité, la conception mä'ohi met l'accent non sur le territoire mais sur un contenu humain, en l'occurrence ici le mata'eina'a.

Un dernier mot, enfin, concernant le phénomène mä’ohi des marae. Bertrand Gérard (1974) a souligné, par l'institution de la pierre de fondation issue d'un édifice existant pour servir de base à la construction d'un nouvel édifice, l'existence de lignées de marae comme il existe des lignées de personnes qui définissent des groupes de descendants; et, par la connexion et le biais des titres de marae portés par des personnages entre des marae et ces derniers, la confortation de ces lignées par des lignées de marae correspondants.

Le « clan » des Teva s'appuie ainsi, selon Tati Salmon (1951) que cite Gérard, dans ses prétentions d'antériorité sur la "famille » royale des Pomare, sur le marae - souche de Vaiotaha à Bora-Bora dédié à Taaroa auquel se trouvent affiliés différents marae dont Farepua à Varari (Papeari) marae des Teva-i-uta plus exactement, i.e. les ari'i de Papara. On a ainsi, à propos d'un « clan », un chaînage qui prétend, de marae en marae, faire descendre un groupe, celui de la famille de Tati Salmon, d'un groupe originaire ancré sur un marae prestigieux. Or, ce faisant, cette reconstruction se présente en contrepoint d'éléments antagonistes, la "famille» Pomare, le dieu 'Oro, le marae Taputapuatea d'Opoa à Raiatea, les duplicata dudit marae à Tahiti et Moorea, éléments disparates qu'elle tend à réorganiser en une construction parallèle concurrente; ce, d'ailleurs, en chahutant la tradition lorsque cela arrange les auteurs : Ariitaimai et ses enfants la reine Marau et Tati Salmon. Pardelà les tentatives de justification de droits révolus, le récit éclaire ce sur quoi reposent les droits des groupes : un chaînage de marae donnant à des chefs des titres sur des terres et leur contenu, potentiel de production et groupe humain.

\section{Espaces en forme de réseau ou pré-carré : la struc- ture spatiale du monde $m \bar{a}$ 'ohi}

Résumons notre propos. L'organisation politique ancienne des $m \bar{a}$ 'ohi est, à l'instar des sociétés polynésiennes, le niveau supérieur de son organisation sociale, elle-même développement de son modèle familial. Celui-ci fut théorisé référence s'impose à Raymond Firth notamment - en termes de ramage, groupe endogame de descendants d'un ancêtre commun donnant naissance à chaque génération à des sousgroupes endogames formés à partir des descendants immédiats de l'ancêtre et ainsi de suite. Comme chacun des descendants à chaque génération se trouve classé par ordre de naissance, chaque sous-groupe d'une même génération se trouve classé selon son rang à l'intérieur du groupe qui l'enveloppe et la société tout entière descendant de l'ancêtre commun voit ses membres rangés par ordre décroissant depuis son chef, aîné vivant du sous-groupe aîné jusqu'au benjamin du benjamin des sous-groupes cadets.

La conséquence est qu'il n'y a pas de rupture dans l'ordre décroissant entre le premier et le dernier de cette société "ramifiée ", ce qui est donc en contradiction avec l'organisation en ordres distincts que l'on rencontre dans les sociétés polynésiennes, sauf à faire intervenir pour son explication des faits de premiers occupants réduits en dépendants du groupe dominant. Chez les Mā'ohi des temps anciens, la société était grossièrement divisée en trois classes (sept niveaux dans la synthèse des sources qu'en fait Gérard, 1974), les hui ari'i (chefs et grandschefs), les hui ra'atira (propriétaires fonciers, commoners par rapport aux hui ari'i), et les manahune (hommes libres pourvus d'une terre d'héritage ou regroupant tous les indépendants, libres ou non selon les districts). Dans la société $m \bar{a}$ ’ohi ancienne, le phénomène central est le marae auquel tout Mā'ohi se réfère pour rendre culte à ses atua (dieux ou esprits) : marae familial, de lignage, de clan, de district ou local, «national» (de groupement de districts ou d'île), « international » (de ligue ou confédération opposant Bora-Bora à Raiatea selon certaines traditions). Pour être plus précis, notons qu'il existe à côté des atua i terai (dieux dans le ciel) d'autres esprits, bénéfiques ou maléfiques, qu'on peut invoquer à des fins magiques positives ou négatives en les priant éventuellement " d'entrer» dans des " images» de pierre ti' $i$ (tiki) pour leur rendre culte et se prêter à des pratiques magiques ou de sorcellerie. Mais tandis que les ti'i relèvent de l'individuel, les marae (c'est en cela qu'ils nous intéressent) sont de l'ordre du social.

Tout Mā'ohi se trouve en principe concerné par les marae des différents groupes auquel il appartient (familial, lignager, clanique, local...). Cela est vrai pour les ari' $i$ admis pour descendre des dieux et porteurs du sacré ( $\mathrm{ra}^{\prime} a$ ) et pour les prêtres ; c'est moins clair pour les gens de condi- 
tion inférieure et qualifiés de profanes (noa) pour lesquels les marae déclarés ra'a sont interdits (tapu). Cette situation provient de la contradiction déjà évoquée entre le principe de ramification qui entraîne la continuité du classement par ordre croissant/décroissant des membres du groupe et l'organisation en classes étanches, contradiction renforcée par les notions de ra'alnoa et tapu. Elle découle aussi, en pratique, de ce que les auteurs qui nous rapportent les traditions ne nous parlent que d'eux-mêmes, ari' $i$, et ne voient les autres, ceux des autres classes, qu'à travers ce qu'ils en savent et selon leur point de vue particulier.

Si l'on s'en tient au pouvoir politique détenu par un ari'i dans son district, ce pouvoir se trouve, tant à l'intérieur (s'exerçant sur les clans et les lignages) qu'à l'extérieur (au sein de confédérations de districts plus ou moins mouvantes), cristallisé en réseaux de marae dont les titulaires (porteurs de titre) sont les subordonnés claniques ou les alter ego du ari'i. Mais rien n'empêche que sur un territoire donné comme un district (fenua), coexistent à côté du réseau clanique du ari'i des éléments de réseaux dépendants d'autres ari'i et donc hors de son propre mata'eina'a. Ainsi pourraient s'expliquer :

- premièrement, la construction par Marama-ite-tauo-o-te-rai ari'i de Haapiti d'une petite structure de marae auprès de Nuurua dans le fenua de Varari, i.e. à deux districts de Haapiti, un marae "national» dont l'ari' $i$ de Varari porte le titre «Punua-i-te-rai-atua» et se trouve donc le supérieur titulaire de Marama ;

- deuxièmement, la possession par Tutaha grand guerrier de Pare à Tahiti du titre du marae Maraetaata, structure du fenua de Paea, peut-être pour des raisons d'alliance de parenté, mais significative éventuellement d'un chaînage de ce marae avec ceux de Pare d'où il est originaire ;

- puis, la localisation dans le même fenua (Paea) de la fameuse " débâcle de Papara » (Oliver, 1974 : III p. 1217) de 1768 opposant les protagonistes du Nord et du Sud complètement étrangers à ce mata'eina' $a$ de l'Ouest membre d'une confédération apparemment neutre en l'espèce, montrant clairement la dissociation entre fenua, territoire, et mata'eina'a, population de l'ari'i localisé sur ce district ;

- enfin, l'érection par Amo, grand-chef de Papara, ari'i maro'ura, d'un marae Taputapuatea dédié à 'Oro à l'embouchure de la rivière Punaruu dans le fenua de Punaauia où se trouve localisé le mata'eina' $a$ du grand-chef de la côte ouest Pohuetea, précisément ari'i rahi (grand ari'i) de cette confédération neutre visà-vis du Nord et du Sud lors de l'événement de 1768.

On voit bien comment des districts de la côte sud-ouest de Moorea pouvaient posséder, sur la carte du capitaine Ribourt (1863), des annexes autre part dans cette île; des groupes partant de ces districts (en raison peut-être de surpeuplement ou de différends...) ont pu s'installer dans des zones libres de population, l'érection de marae issus par leur pierre de fondation des marae des districts d'origine suffisant pour ancrer ces groupes dans leur nouveau paysage. La localisation à Maeva, à Huahine, des marae têtes des différents « clans » de l'île sous la suprématie en quelque sorte du grand marae Matairea permet de supposer des chaînages de marae égrenant chaque clan en divers lieux de l'île dans un ensemble spatial « réticulé », en "système de fils croisés » (lat. reticulum " petit filet», de rete « rets », selon le Nouveau petit Robert, 1993).

Si une telle structure en chaînage, d'espaces en réseaux, réticulaire, n'apparaît pas dans les discours $m \bar{a}$ 'ohi ou européens qui disent la tradition ancienne, c'est peut-être que cette structure était au moment de la « découverte » en voie d'abandon au profit d'une territorialisation des espaces sociaux, susceptible au niveau des districts de faire coïncider fenua et mata'eina'a. Mais, c'est peut-être aussi que le concept européen de gouvernement sur un territoire défini par ses limites et coïncidant avec son contenu humain que les interrogateurs européens de la tradition avaient en tête, n'a pas permis à ces derniers de poser les bonnes questions à propos d'une conception $m \bar{a}$ 'ohi de l'espace qui n'était plus peut-être, dans le monde $m \bar{a}$ 'ohi d'alors, d'actualité.

\section{BIBLIOGRAPHIE}

Adams Henry, 1964. Mémoires d'Ariitaimai, Paris, Société des Océanistes, Publications de la Société des Océanistes 12.

Bensa Alban et Jean-Claude RiviERre, 1982. Les chemins de l'alliance, Paris, Selaf, coll. Langues et cultures du Pacifique 1.

Bonnemaison Joël, 1996. Gens de la pirogue et gens de la terre. Les fondements géographiques d'une identité : l'archipel du Vanuatu. Essai de géographie culturelle: Livre I, Paris, onstom éditions ( $1^{\text {ère }}$ éd. 1986, Les fondements d'une identité : Territoire, histoire et société dans l'archipel du Vanuatu [Mélanésie]. Livre I, L'arbre et la pirogue, Paris, ORSTOM éditions). 
Davies J, 1961. The History of Tahitian Mission 17991830, Cambridge, Cambridge University Press, The Hakluyt Society, $2^{\text {nd }}$ ser., CXVI.

EMORY Kenneth P., 1932 Traditional History of Maraes in the Society Islands, Honolulu, Hawaii, B.P. Bishop Museum, unpublished manuscript.

FIRTH Raymond, 1936. We The Tikopia. A Sociological Study of Kindship in Primitive Polynesia, London, George Allen and Unwin Ltd.

-, 1965. Primitive Polynesian Economy, London, Routledge and Kegan Paul.

Garanger José, 1969. Pierres et rites sacrés du Tahiti d'autrefois, Paris, Société des Océanistes, Dossiers tahitiens 2.

—, 1972. Archéologie des Nouvelles-Hébrides. Contribution à la connaissance des îles du Centre, Paris, Société des Océanistes-orstom, Publications de la Société des Océanistes 30.

GÉRARD Bertrand, 1974. Origine traditionnelle et rôle social des marae aux îles de la Société, Cahiers de l'ORSTOM, série sciences humaines 11, 3-4, pp. 211226.

Guiart Jean, 1963. Structure de la chefferie en Mélanésie du Sud, Paris, musée de l'Homme, université de Paris, Travaux de l'Institut d'Ethnologie LXVI.

HANDy Edward Smith Craighill, 1930. History and Culture in the Society Islands, Honolulu, Hawaii, B.P. Bishop Museum Bulletin 79.

Henry Teuira, 1962. Tahiti aux temps anciens, Paris, Société des Océanistes, Publications de la Société des Océanistes 1 (traduction de Ancient Tahiti, B.P. Bishop Museum, Honolulu, Hawaii, 1928).

Marau Taaroa, 1971. Mémoires de Marau Taaroa, dernière reine de Tahiti, traduits par sa fille la princesse Ariimanihini Takau Pomare, Paris, Société des Océanistes, Publications de la Société des Océanistes 27.

NAtua Aurora, directrice du musée de Papeete, communication personnelle, 1967-1968.

OLIVER Douglas, 1974. Ancient Tahitian Society, Honolulu, Hawaii, The University Press of Hawaii, 3 vol.

RIBOURT Pierre, 1863. État de l'île de Tahiti durant les années 1847-1848, Annuaire de Tahiti, Papeete, pp. 302-324 ( $1^{\text {ère }}$ publication, 1850, Revue coloniale, Paris).

Robineau Claude, 1998. Chefferie, leadership et communautés territoriales aux îles de la Société, in D. Guillaud, M. Seysset et A. Walter (éds), Le voyage inachevé : À Joël Bonnemaison, Paris, Éd. de l'ORSTOM et PRODIG, UMR I63, CNRS, pp. 319-323.

Rodriguez Maximo, 1995. Les Espagnols à Tahiti 1772-1774, Journal de Maximo Rodriguez, traduit et annoté par Horacio Belçaguy, Paris, Société des Océanistes, Publications de la Société des Océanistes 45.

SAHLIns Marshall, 1958. Social Stratification in Polynesia, Seattle, University of Washington Press, American Anthropological Monograph Series, M 29.

Salmon Tati, 1951. L'histoire de l'île de Bora-Bora et la généalogie de notre famille du marae Vaiotaha, Bulletin de la Société d'études océaniennes VIII, 97, pp. 315-330 (manuscrit de 1904 traduit de l'anglais).

TERII-VAHINE-I-TE-TAUO-O-TE-RAI， 1970， Marama, famille puissante et très ancienne des Mers du Sud, Bulletin de la Société d'études océaniennes XIV, 11 et 12, pp. $172-173$ et pp. $397-400$. 
